\title{
La educación ambiental en la formación de profesores en Chile
}

\author{
The environmental education in the training of teacher's in Chile
}

\author{
FRANKLIN CASTILLO-RETAMAL \\ Facultad de Ciencias de la Educación \\ Universidad Católica del Maule, Talca, Chile \\ fcastillo@ucm.cl
}

\section{FERNANDA CORDERO-TAPIA}

Facultad de Ciencias de la Educación

Universidad Católica del Maule, Talca, Chile

fdacorderotapia@gmail.com

\section{RESUMEN}

objetivo de este estudio es entregar una visión sinóptica acerca del recorrido y la presencia que ha tenido la educación ambiental en el currículum escolar y en la formación de profesores de educación básica en Chile. Para ello, se realizó una revisión bibliográfica y documental. Los datos se analizaron a partir de su contenido. Los resultados indican que existe cierta preocupación para incorporar la educación ambiental en el currículo escolar, así como en los proyectos formativos universitarios, evidenciándose un abordaje parcial de la misma. Como conclusión, se refleja que existe una voluntad de acercamiento a la temática ambiental, pero es necesario que esta se enfatice aún más, a fin de alcanzar mejores resultados.

Palabras clave: Formación de profesores, educación ambiental, currículo, escuela.

\section{ABSTRACT}

The aim of this study is to provide a synoptic view of the route and presence of environmental education in the school curriculum and in the training of basic education teachers in Chile. For this, a bibliographic and documentary review was carried out. The data was analyzed based on its content. The results indicate that there is some concern for the incorporation of environmental education in the school curriculum, as well as in university training projects, evidencing a partial approach to it. As a conclusion, it is evident that there is a willingness to approach the environmental issue, but it is necessary that it be emphasized even more, in order to achieve better results.

Key words: Training teacher's, environmental education, curriculum, school. 


\section{Introducción}

Desde los inicios de la humanidad, el hombre ha tenido una estrecha relación con el medio en el que vive, aprovechando lo que la tierra le provee e interactuando con ella, incluso sintiéndose con pleno derecho para explotar sus bienes y recursos, lo que provocó una aceleración de los procesos de degradación del medio ambiente. Es indiscutible la acción nociva del hombre.

Entre las causas que han provocado el deterioro del planeta se encuentran: el desarrollo social, científico y tecnológico, las estructuras políticas, el estilo de vida del mundo moderno basado en el consumo, la cultura del "usar y tirar" y la búsqueda de una calidad de vida consistente en acumular bienes.

Por otro lado, si bien en las últimas décadas se establecieron diferentes acuerdos internacionales, pactos y tratados para dar respuestas favorables a las problemáticas ambientales, es posible apreciar que los avances en cuanto a contaminación no han sido significativos, por lo que se requiere una modificación conductual y una nueva actitud hacia el medio.

En ese sentido, la educación ambiental (EA) cumple un rol importante en la toma de conciencia del individuo, pues proporciona nuevos conocimientos y facilita la comprensión de los problemas que afectan al medio ambiente, junto con ello, es una herramienta de alta importancia para afrontar la tarea de provocar un cambio en las conductas y hábitos en relación a la naturaleza y los elementos que la componen (Fuentealba y Soto, 2016).

Al respecto, la educación cumple un rol fundamental al propiciar -desde las escuelas y los diferentes centros educativos- acciones para favorecer en las nuevas generaciones- el desarrollo de hábitos amigables con el medio ambiente, a fin de modificar las conductas erróneas. En todo caso, "el fomento de la responsabilidad requiere de un fuerte impulso de la EA en el ámbito formal y no formal que favorezca y promueva buenas prácticas ambientales" (Castillo y Fuentealba, 2014, p. 8).

Sin embargo, la educación por sí sola no consigue resolver la totalidad de los problemas ambientales que son atribuibles a factores económicos, sociales, físicos, ecológicos, biológicos y culturales, aunque con el auxilio y refuerzo de la ciencia y tecnología, es posible que contribuya a formular algunas soluciones basadas en la solidaridad y equidad (UNESCO, 1980). 
Desde esa perspectiva, el docente no se considera un emisor de conocimientos, sino que se convierte en un agente sensibilizador, capaz de provocar en los estudiantes cambios de conductas y hábitos hacia el medio ambiente.

La formación del profesorado resulta un factor clave para la integración de la EA en el sistema educativo formal, pues si el docente no conoce qué es, ni en qué consiste su práctica, difícilmente podrá incorporarlo a su labor en el aula (Martín, 1996).

En Chile, el Ministerio de Educación adaptó el curriculum siguiendo la línea de las definiciones curriculares de 1996, que se mantuvieron en las actualizaciones de 2009, cuando se elaboran las Bases Curriculares para la Educación Básica (Chile, 2009), momento en que la EA se presenta de manera transversal en el curriculum escolar. Aun así, que esta se desarrolle como tal o que se tome en cuenta en los diseños escolares, depende posiblemente de la formación de los profesionales de la educación en esta área y del tratamiento que se le da en su proceso de formación inicial.

En este sentido, el cambio de hábitos y de conductas -desde los objetivos que plantea la EA- se llevará a cabo desde la educación y a partir de la forma en la que se estén desarrollando esos aspectos en los procesos de formación docente, tal como lo destaca la UNESCO (1977), al sostener que la posibilidad de integrar la EA en la enseñanza formal y no formal depende principalmente de la formación de aquellos que deben llevar a cabo estos programas.

Es decir, se considera que la educación -como agente de transformación social- tiene un rol preponderante en la generación y promoción de cambios, a través del desarrollo de habilidades blandas y habilidades cognitivas (Fuentealba, Marín, Castillo y Roco, 2017).

Por lo antes expuesto, con este trabajo se busca atender al menos dos objetivos: 1) establecer una visión panorámica con respecto de la $E A$, a partir de su evolución y tratamiento; y, 2) presentar el abordaje de la EA en la formación de profesores de enseñanza básica en Chile. Para esto, se utilizó la metodología cualitativa básica, pues se pretenden generar conocimientos nuevos y útiles, sin una inmediata aplicación práctica (Engel y Tolfo, 2009). Como procedimiento, se adoptó el análisis de documentos y la investigación bibliográfica, elaborados a partir de materiales publicados, pero que no poseen un tratamiento analítico (Silva y Muskat, 2005). 
La selección de la literatura, que atiende al primer objetivo, se tomó de las bases de datos: Scopus, Scielo, Google Académico y Latindex. Mientras que, entre los documentos se analizaron los programas de estudio de las carreras de pedagogía básica de las universidades pertenecientes al Consejo de Rectores, así como leyes, decretos, documentos oficiales y no oficiales.

\section{Una aproximación al concepto de educación ambiental}

En la primera reunión internacional sobre medio ambiente, convocada por la UNESCO (1972) y celebrada en Estocolmo bajo el título de "Conferencia de las Naciones Unidas sobre el medio ambiente humano", se concluyó que resulta indispensable desarrollar una educación en cuestiones ambientales, la cual debe estar "dirigida tanto a las generaciones jóvenes como a los adultos y que preste la debida atención al sector de población menos privilegiado, para ensanchar las bases de una opinión pública bien informada" (UNESCO, 1973, p. 5)

Asimismo, en la "Conferencia intergubernamental de educación ambiental", desarrollada en Tbilisi (1977), se examinaron los principales problemas de la humanidad y se determinó el papel que podía tener la educación en su resolución. En esa oportunidad, se definió la EA como:

"El proceso a través del cual se aclaran los conceptos sobre los procesos que suceden en el entramado de la naturaleza, se facilitan la comprensión y valoración del impacto de las relaciones entre el hombre, su cultura y los procesos naturales y sobre todo se alienta a un cambio de valores, actitudes y hábitos que permitan la elaboración de un código de conducta con respecto a las cuestiones relacionadas con el medio ambiente".

Esa definición consideró la perspectiva social de la EA al mencionar las relaciones sociales, cultura y valores. Esos dos componentes -ambiental y socialhan aparecido desde el comienzo en las definiciones de EA, pero con los años cobró más relevancia el aspecto social (Alea, 2005).

Por otro lado, en el Congreso Internacional de Educación y Formación sobre el Medio Ambiente (Moscú, 1987) se propuso la definición de EA como:

"Un proceso permanente en el cual los individuos y las comunidades adquieren conciencia de su medio y aprenden los conocimientos, los valores, las destrezas, la experiencia y también la determinación que les 
capacite para actuar individual y colectivamente en la resolución de los problemas ambientales presentes y futuros".

Otra conceptualización de la EA es la desarrollada en el Foro Global de la Sociedad Civil (Río de Janeiro, 1992), donde se considera que la EA debe ser para una sociedad sostenible y equitativa:

"Un proceso de aprendizaje permanente, basado en el respeto a todas las formas de vida. Tal educación afirma valores y acciones que contribuyen a la transformación humana y social con el fin de conseguir la preservación ecológica. También la Educación Ambiental estimula la formación de sociedades socialmente justas y ecológicamente equilibradas, que establecen relaciones entre sí de interdependencia y diversidad. Esto requiere responsabilidad individual y colectiva a nivel local, nacional y planetario".

Al respecto, resulta innegable que el concepto de EA evoluciona según lo que sucede con el medio ambiente y en la sociedad en un momento determinado. De esa manera, si bien en un inicio la importancia recaía sobre el mismo medio, con el pasar de los años se deja de manifiesto que el aspecto social tomó más protagonismo, por lo que se la da más énfasis a la transversalidad de la EA.

Martínez (1999) plantea que:

"Actualmente sabemos que, aunque lo físico-natural es la base de nuestro medio, las dimensiones socioculturales, políticas y económicas son fundamentales y proporcionan instrumentos conceptuales para comprender mejor las relaciones que la humanidad establece con su medio y para gestionar mejor los recursos naturales".

Por su parte, Echarri (2007) destaca que las diversas definiciones de EA coinciden en algunos aspectos que, de una u otra manera, conllevan al objetivo principal de la educación ambiental, tal como se aprecia en la Figura 1: 


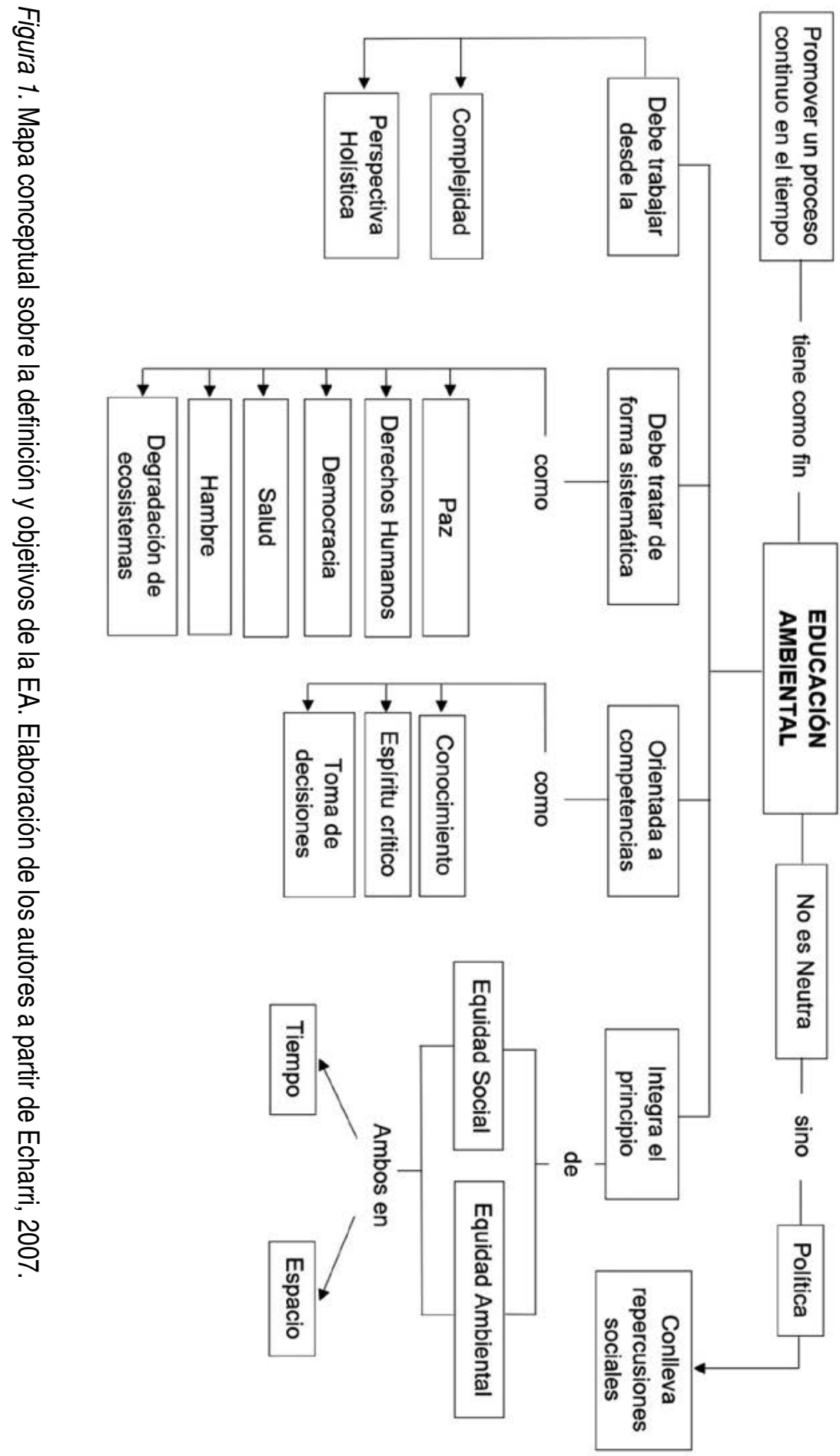




\section{Los antecedentes históricos de la educación ambiental}

El desarrollo del término EA surge a finales de la década de los años 60 y principios de los años 70. En la tabla 1 se muestran los hitos más importantes en relación a la $E A$, en los cuales se formularon los parámetros que sustentan la educación ambiental:

\section{Tabla 1}

Antecedentes históricos de la educación ambiental.

\begin{tabular}{|c|c|c|c|}
\hline Año & Evento & País & Resultados/productos \\
\hline 1972 & $\begin{array}{l}\text { Conferencia de las } \\
\text { Naciones Unidas } \\
\text { sobre el Medio } \\
\text { Humano }\end{array}$ & $\begin{array}{c}\text { Suecia } \\
\text { (Estocolmo) }\end{array}$ & $\begin{array}{l}\text { - Se establece el concepto de } \\
\text { educación ambiental. } \\
\text { - Se crea el Programa de las } \\
\text { Naciones Unidas para el Medio } \\
\text { Ambiente. }\end{array}$ \\
\hline 1975 & $\begin{array}{l}\text { Seminario } \\
\text { Internacional } \\
\text { de Educación } \\
\text { Ambiental }\end{array}$ & $\begin{array}{l}\text { Yugoslavia } \\
\text { (Belgrado) }\end{array}$ & $\begin{array}{l}\text { En este evento surge la Carta } \\
\text { de Belgrado, que fija las metas } \\
\text { y los objetivos de la educación } \\
\text { ambiental. Asimismo, se } \\
\text { delimitan sus contenidos. }\end{array}$ \\
\hline 1977 & $\begin{array}{l}\text { Conferencia } \\
\text { Intergubernamental }\end{array}$ & $\begin{array}{l}\text { URSS } \\
\text { (Tbilisi) }\end{array}$ & $\begin{array}{l}\text { En la Declaración de Tbilisi se } \\
\text { establecieron los lineamientos, } \\
\text { criterios y directrices que habrían } \\
\text { de inspirar todo el desarrollo del } \\
\text { movimiento educativo en las } \\
\text { décadas posteriores. }\end{array}$ \\
\hline 1987 & $\begin{array}{l}\text { Congreso } \\
\text { Internacional de } \\
\text { Moscú }\end{array}$ & $\begin{array}{l}\text { URSS } \\
\text { (Belgrado) }\end{array}$ & $\begin{array}{l}\text { - Se acuerda declarar la década } \\
\text { de los noventa como la "Década } \\
\text { mundial para la educación } \\
\text { ambiental". } \\
\text { - } \quad \text { Los trabajos se organizaron en } \\
\text { torno a elementos decisivos } \\
\text { de la EA y se insistió en la } \\
\text { necesidad de hacer énfasis en } \\
\text { un desarrollo sustentable. }\end{array}$ \\
\hline 1992 & $\begin{array}{l}\text { Conferencia de } \\
\text { las Naciones } \\
\text { Unidas sobre } \\
\text { Medio Ambiente y } \\
\text { Desarrollo }\end{array}$ & $\begin{array}{c}\text { Brasil } \\
\text { (Río de Janeiro) }\end{array}$ & $\begin{array}{l}\text { - Se establece la llamada Agenda } \\
21 .\end{array}$ \\
\hline
\end{tabular}




\begin{tabular}{|c|c|c|c|}
\hline Año & Evento & País & Resultados/productos \\
\hline 1992 & $\begin{array}{l}\text { Congreso } \\
\text { Iberoamericano } \\
\text { de Educación } \\
\text { Ambiental }\end{array}$ & $\begin{array}{c}\text { México } \\
\text { (Guadalajara) }\end{array}$ & $\begin{array}{l}\text { - Contextualización de la EA para } \\
\text { América Latina para el logro del } \\
\text { desarrollo sostenible. }\end{array}$ \\
\hline 1995 & $\begin{array}{l}\text { I Conferencia de } \\
\text { las Partes para la } \\
\text { Convención sobre } \\
\text { Cambio Climático }\end{array}$ & $\begin{array}{l}\text { Alemania } \\
\text { (Berlín) }\end{array}$ & $\begin{array}{l}\text { - Se concluye que el abordaje } \\
\text { escogido fracasó. } \\
\text { - La Conferencia resulta en el } \\
\text { mandato de Berlín, que hace } \\
\text { un llamado a las naciones más } \\
\text { industrializadas a establecer } \\
\text { objetivos específicos para la } \\
\text { reducción de sus emisiones. }\end{array}$ \\
\hline 1997 & $\begin{array}{l}\text { Conferencia } \\
\text { Internacional } \\
\text { Medio Ambiente } \\
\text { y Sociedad: } \\
\text { Educación y } \\
\text { Sensibilización del } \\
\text { Público }\end{array}$ & $\begin{array}{c}\text { Grecia } \\
\text { (Tesalónica) }\end{array}$ & $\begin{array}{l}\text { - Educación para un futuro } \\
\text { sostenible. }\end{array}$ \\
\hline 2002 & $\begin{array}{l}\text { Cumbre Mundial } \\
\text { sobre el Desarrollo } \\
\text { Sostenible }\end{array}$ & $\begin{array}{c}\text { Sudáfrica } \\
\text { (Johannesburgo) }\end{array}$ & $\begin{array}{l}\text { Educación para el desarrollo } \\
\text { sostenible. }\end{array}$ \\
\hline
\end{tabular}

Según Calderón, Sumarán, Chumpitaz y Campos (2011), uno de los propósitos de la EA es que la comunidad comprenda la naturaleza compleja del ambiente y, a partir de ello, adquirir "conocimientos, valores y habilidades prácticas para participar en la prevención y solución de problemas ambientales" (p. 21). En tal sentido, indican también que es necesario dotar a los individuos de las herramientas que les permitan desarrollar y profundizar en la información a través de la investigación y el análisis, de tal forma que consigan comprender la problemática ambiental; desarrollar las capacidades necesarias para dar solución a problemas actuales y futuros y; desarrollar habilidades que garanticen un proceso educativo adecuado y continuo.

Ahora bien, durante el Seminario Internacional de EA celebrado en octubre de 1975 en Belgrado, se establecieron los objetivos generales de la EA en la denominada Carta de Belgrado, sin embargo, autores como Picón y Mendoza (2011) sostienen que las propuestas en materia de EA deben tener como objetivos lo que se plantean en la Figura 2: 


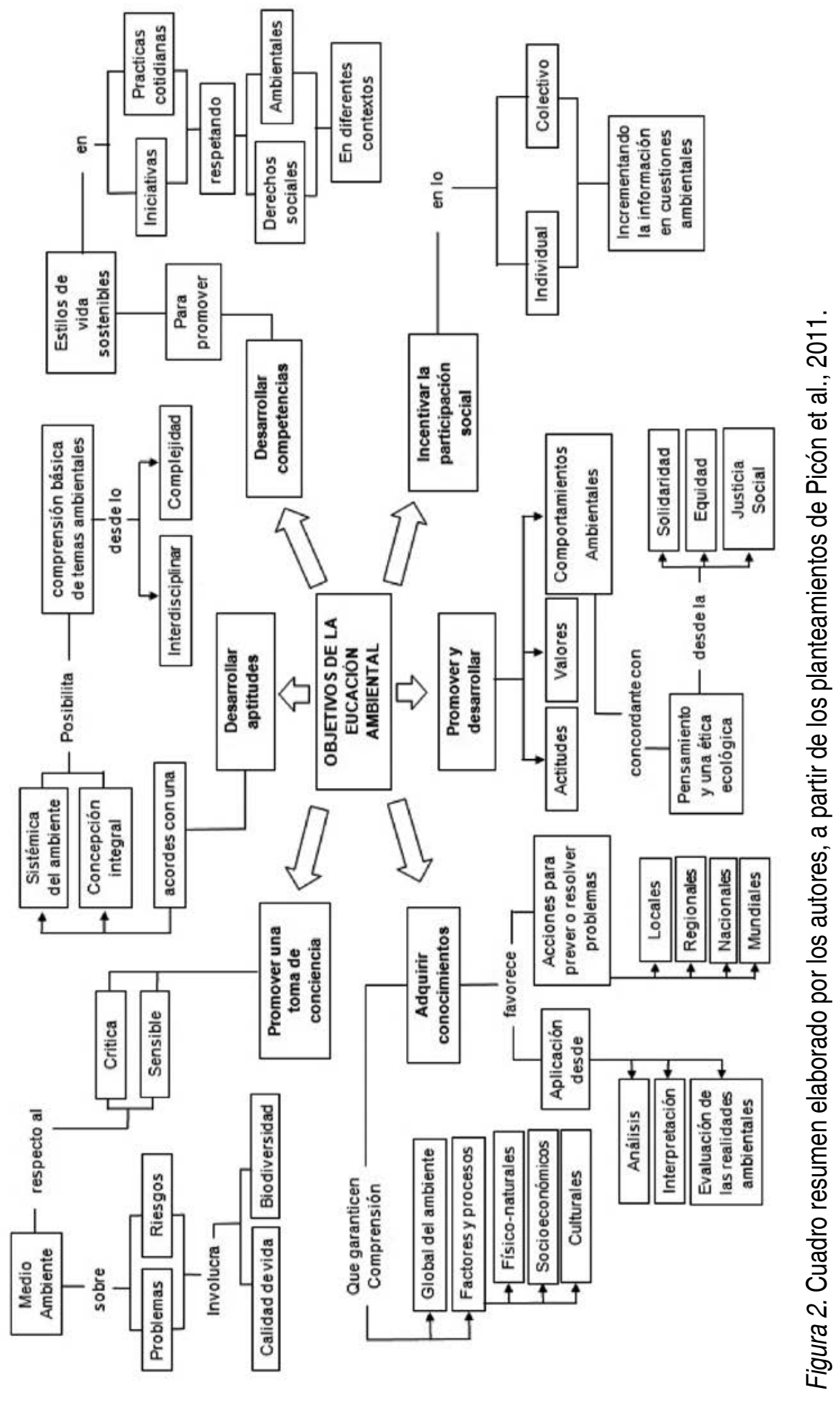

UCMaule - Revista Académica N56 - Junio 2019 


\section{Educación ambiental en la formación inicial de profesores en Chile}

En 1983, el ministro de Agricultura -a instancias de la Corporación Nacional Forestal (CONAF)- sugirió al ministro de Educación realizar un programa de EA. Como resultado, se elaboró el documento "Estrategia de ejecución del Programa de Educación Ambiental", en el que se describió el proyecto a nivel nacional denominado "Ciencias Integradas Básicas Experimentales" (CIBEX), que propendía al mejoramiento de la enseñanza y aprendizaje de las ciencias naturales.

Posteriormente, en 1984, con el propósito de dar algunas directrices para el desarrollo de una conducta ambiental a los ciudadanos chilenos, la Universidad Metropolitana de Ciencias de la Educación editó el libro "Educación Ambiental: Hacia el desarrollo de una conducta ecológica en Chile". La publicación estuvo dirigida, principalmente, a docentes de enseñanza básica y media (Omegna, 2003).

En 1990, se plantea en el programa de gobierno un capítulo que sugiere la formulación de una política ambiental nacional, a fin de afrontar los problemas de los centros urbanos en términos de la contaminación del agua y del aire, principalmente (Omegna, 2003), con todo, hasta el momento no ha sido posible estructurar en el país una política en EA de manera explícita (Muñoz, 2014). No obstante aquello, existe un acercamiento a partir de las definiciones que la Ley de Bases del Medio Ambiente (Ley № 19.300 de 1994) tiene a disposición de la comunidad, conceptualización que fue utilizada como referencia en este trabajo y son descritas como:

- "Educación Ambiental: es un proceso permanente de carácter interdisciplinario destinado a la formación de una ciudadanía que reconozca valores, aclare conceptos y desarrolle las habilidades y las actitudes necesarias para una convivencia armónica entre seres humanos, su cultura y su medio bio-físico circundante".

- "Medio Ambiente: es el sistema global, constituido por elementos naturales y artificiales de naturaleza física, química o biológica, socioculturales y sus interacciones, en permanente modificación por la acción humana 0 natural y que rige y condiciona la existencia y desarrollo de la vida en sus múltiples manifestaciones". 
En 1990 el Ministerio de Educación, a razón de las directrices del programa de gobierno de la época, tiene como objetivo la incorporación de la EA en el currículo y así adquiera una mayor importancia. En este sentido, la Ley Orgánica Constitucional de Enseñanza de 1990 (LOCE), propone como objetivo transversal este tema y se condice en las acciones relativas a la elaboración de material educativo, asesorías técnicas y apoyo a las escuelas en términos financieros. Por otro lado, la Ley 19.300 (Chile, 1994) propone la obligatoriedad de la EA en el sistema educacional. A partir de esta Ley, se crea una entidad denominada Comisión Nacional de Medio Ambiente (CONAMA), que se encargará de desarrollar las políticas públicas en materia de medioambiente en el país (Castillo, 2011).

Es importante resaltar que, además, la Constitución Política de la República de Chile establece en su artículo 19, № 8 en su última versión (Chile, 2018): "el derecho a vivir en un medio ambiente libre de contaminación. Es deber del Estado velar para que este derecho no sea afectado y tutelar la preservación de la naturaleza". Es decir que, considerando ese planteamiento, vivir en un ambiente sin contaminación es un derecho constitucional, lo que deberá ser asegurado por el Estado, por lo que la EA puede ser entendida como una forma de velar por el cumplimiento del artículo mencionado.

Aunado a ello, la Ley de Bases del Medio Ambiente (Chile, 1994 Chile, 1994 y última versión de junio de 2016), otorgó a la educación la responsabilidad de ser un instrumento de gestión ambiental, por lo tanto, la educación asume un rol preponderante en torno a la formación de ciudadanos ambientalmente responsables de lo que sucede y de lo que no sucede en el escenario en el que están insertos.

Para lograr esa formación, la EA debe ser entendida como un proceso pedagógico con una mirada sistémica del ser humano, por lo que se deben desarrollar planos como el: a) intelectual, al ofrecer contenidos temáticos que sienten las bases teóricas al respecto; b) práctico, al promover y desarrollar acciones para el mejoramiento y cuidado de los recursos, a fin de asegurar su disponibilidad tanto en calidad como en cantidad; y c) ético-moral, al sopesar los significados que conllevan los contenidos, considerando la formación en valores, la cual requiere una instrucción mediada por el profesor, a través de la implementación de estrategias y de la organización de actividades para promover en los estudiantes la formación de aquellos aspectos relevantes que se enfatizan en los objetivos transversales propuestos por el marco curricular vigente, pudiendo 
utilizar y transferir los aprendizajes a otras situaciones, por lo tanto, la formación de los profesores en estas áreas es fundamental (Castillo y Almonacid, 2012).

Por lo antes expuesto, se puede afirmar que, en el caso de las universidades chilenas, el abordaje de la EA resulta un área poco tratada en los programas de pedagogía. Al respecto, si bien desde la década de 1970 se incorporaron algunos aspectos relacionados con temáticas del medio ambiente en los cursos del currículo de formación de profesores, estos son de carácter optativo, pues el desarrollo de las temáticas medioambientales queda supeditada a la subjetividad y motivación del profesor responsable de las asignaturas del plan de formación profesional.

Por otro lado, el Consejo de Rectores de Universidades Chilenas (CRUCH) está constituido por 25 universidades, de las cuales 19 imparten la carrera de Pedagogía General Básica. En la revisión de las mallas curriculares de esta carrera, solo 16 universidades plantean asignaturas alusivas a las ciencias naturales o comprensión del medio, pero ello no implica que dentro del abordaje de la asignatura se consideren temas alusivos a la EA.

Es decir, esas universidades incorporan la EA como tema 0 abordan algún contenido específico de esta en las cátedras, pero no se asume la EA como asignatura, ni mucho menos es vinculada con otras asignaturas de las mallas curriculares. Lo anterior deriva en el hecho de que la EA siga siendo uno de los contenidos invisibles en los programas de estudio; esto podría develar que la EA no se trabaje de manera interdisciplinar o trasversal. 


\section{Tabla 2}

La educación ambiental en las mallas curriculares de la carrera de Pedagogía General Básica en las universidades del CRUCH.

\begin{tabular}{|c|c|c|c|}
\hline $\mathbf{N}^{\circ}$ & UNIVERSIDAD & CARRERA & ASIGNATURAS \\
\hline 1 & $\begin{array}{l}\text { Universidad de } \\
\text { Chile }\end{array}$ & & \\
\hline 2 & $\begin{array}{l}\text { Pontificia } \\
\text { Universidad } \\
\text { Católica de Chile }\end{array}$ & $\begin{array}{l}\text { Pedagogía General Básica con } \\
\text { mención en Ciencias Naturales. } \\
\text { Pedagogía General Básica con } \\
\text { mención en Ciencias Sociales. } \\
\text { Pedagogía General Básica } \\
\text { con mención en Lenguaje y } \\
\text { Comunicación. } \\
\text { Pedagogía General Básica con } \\
\text { mención en Matemática. }\end{array}$ & $\begin{array}{ll}\text { - } & \text { Didáctica de las } \\
\text { Ciencias Naturales I. } \\
\text { - } \text { Didáctica de las } \\
\text { Ciencias Naturales II. }\end{array}$ \\
\hline 3 & $\begin{array}{l}\text { Universidad de } \\
\text { Concepción }\end{array}$ & $\begin{array}{l}\text { Educación Básica, especialista } \\
\text { en Matemática. } \\
\text { Educación Básica, especialista } \\
\text { en Ciencias Naturales. }\end{array}$ & $\begin{array}{l}\text { - Clasificación de los } \\
\text { Seres Vivos y su } \\
\text { Ambiente. } \\
\text { - Ecología y } \\
\text { Comunidad. } \\
\text { - Didáctica de las } \\
\text { Ciencias Naturales. }\end{array}$ \\
\hline 4 & $\begin{array}{l}\text { Pontificia } \\
\text { Universidad } \\
\text { Católica de } \\
\text { Valparaíso }\end{array}$ & $\begin{array}{l}\text { Pedagogía en Educación } \\
\text { Básica. }\end{array}$ & $\begin{array}{l}\text { - No se evidencia } \\
\text { asignatura relativa a la } \\
\text { EA. }\end{array}$ \\
\hline 5 & $\begin{array}{l}\text { Universidad } \\
\text { Técnica Federico } \\
\text { Santa María }\end{array}$ & & \\
\hline 6 & $\begin{array}{l}\text { Universidad de } \\
\text { Santiago de Chile }\end{array}$ & $\begin{array}{l}\text { Profesor de Educación General } \\
\text { Básica con Mención en } \\
\text { Matemática y Comprensión del } \\
\text { Medio Natural. } \\
\text { Profesor de Educación } \\
\text { General Básica con Mención } \\
\text { en Lenguaje, Comunicación y } \\
\text { Comprensión del Medio Social. }\end{array}$ & $\begin{array}{l}\text { - Estudio y } \\
\text { Comprensión de la } \\
\text { Naturaleza I. } \\
\text { - Estudio y } \\
\text { Comprensión de la } \\
\text { Naturaleza II. } \\
\text { - Estudio y } \\
\text { Comprensión de la } \\
\text { Naturaleza III. } \\
\text { - Didáctica y Evaluación } \\
\text { de Estudio y } \\
\text { Comprensión de la } \\
\text { Naturaleza. }\end{array}$ \\
\hline
\end{tabular}




\begin{tabular}{|c|c|c|c|}
\hline $\mathrm{N}^{\circ}$ & UNIVERSIDAD & CARRERA & ASIGNATURAS \\
\hline 7 & $\begin{array}{l}\text { Universidad Austral } \\
\text { de Chile }\end{array}$ & $\begin{array}{l}\text { Pedagogía en Educación } \\
\text { Básica. }\end{array}$ & $\begin{array}{l}\text { - Ambiente y Seres } \\
\text { Vivos / Módulo: } \\
\text { Enseñanza y } \\
\text { Aprendizaje de las } \\
\text { Ciencias Naturales. }\end{array}$ \\
\hline 8 & $\begin{array}{l}\text { Universidad } \\
\text { Católica del Norte }\end{array}$ & $\begin{array}{l}\text { Pedagogía en Educación } \\
\text { Básica con Especialización. }\end{array}$ & $\begin{array}{ll}\text { - } & \text { Ciencias Naturales I. } \\
\text { - } & \text { Didáctica de las } \\
& \text { Ciencias Naturales II. } \\
\text { - } & \text { Didáctica de las } \\
& \text { Ciencias Naturales III. }\end{array}$ \\
\hline 9 & $\begin{array}{l}\text { Universidad de } \\
\text { Valparaíso }\end{array}$ & & \\
\hline 10 & $\begin{array}{l}\text { Universidad } \\
\text { Metropolitana de } \\
\text { Ciencias de la } \\
\text { Educación }\end{array}$ & $\begin{array}{l}\text { Educación Básica con Mención } \\
\text { en Matemática. } \\
\text { Educación Básica con Mención } \\
\text { en Lenguaje y Comunicación. } \\
\text { Educación Básica con Mención } \\
\text { en Ciencias Naturales. } \\
\text { Educación Básica con Mención } \\
\text { en Ciencias Sociales. } \\
\text { Educación Básica con Mención } \\
\text { en Primer Ciclo. }\end{array}$ & $\begin{array}{l}\text { - Conocimiento y } \\
\text { Comprensión del } \\
\text { Medio Natural y su } \\
\text { Enseñanza I. } \\
\text { - Conocimiento y } \\
\text { Comprensión del } \\
\text { Medio Natural y su } \\
\text { Enseñanza II. } \\
\text { - Conocimiento y } \\
\text { Comprensión del } \\
\text { Medio Natural y su } \\
\text { Enseñanza III. } \\
\text { - Desarrollo } \\
\text { Sustentable. }\end{array}$ \\
\hline 11 & $\begin{array}{l}\text { Universidad } \\
\text { Tecnológica } \\
\text { Metropolitana }\end{array}$ & & \\
\hline 12 & $\begin{array}{l}\text { Universidad de } \\
\text { Tarapacá }\end{array}$ & $\begin{array}{l}\text { Pedagogía en Educación } \\
\text { Básica. }\end{array}$ & $\begin{array}{l}\text { - Introducción a las } \\
\text { Ciencias Naturales. } \\
\text { - Ciencias Naturales } \\
\text { Básica I. } \\
\text { - } \quad \text { Ciencias Naturales } \\
\text { Básica II. }\end{array}$ \\
\hline 13 & $\begin{array}{l}\text { Universidad } \\
\text { Arturo Prat }\end{array}$ & $\begin{array}{l}\text { Pedagogía en Educación } \\
\text { Básica con Mención en } \\
\text { Lenguaje y Ciencias Sociales, } \\
\text { Matemática y Ciencias } \\
\text { Naturales. }\end{array}$ & $\begin{array}{l}\text { - } \\
\text { No se evidencia } \\
\text { asignatura relativa la } \\
\text { EA. }\end{array}$ \\
\hline
\end{tabular}




\begin{tabular}{|c|c|c|c|}
\hline $\mathbf{N}^{\circ}$ & UNIVERSIDAD & CARRERA & ASIGNATURAS \\
\hline 14 & $\begin{array}{l}\text { Universidad de } \\
\text { Antofagasta }\end{array}$ & $\begin{array}{l}\text { Pedagogía Educación General } \\
\text { Básica. }\end{array}$ & $\begin{array}{l}\text { - Estructura y Función } \\
\text { de los Seres Vivos. } \\
\text { - La Tierra, el Universo } \\
\text { y su Interacción con el } \\
\text { Medio. } \\
\text { - Taller de Enseñanza } \\
\text { Aprendizaje de } \\
\text { Ciencias Naturales. }\end{array}$ \\
\hline 15 & $\begin{array}{l}\text { Universidad de } \\
\text { La Serena }\end{array}$ & $\begin{array}{l}\text { Pedagogía en Educación } \\
\text { General Básica }\end{array}$ & $\begin{array}{l}\text { - Comprensión del } \\
\text { Medio Natural I. } \\
\text { - Comprensión del } \\
\text { Medio Natural II. } \\
\text { - } \quad \text { Metodología de la } \\
\text { Enseñanza de las } \\
\text { Ciencias Naturales. }\end{array}$ \\
\hline 16 & $\begin{array}{l}\text { Universidad de } \\
\text { Playa Ancha }\end{array}$ & $\begin{array}{l}\text { Pedagogía en Educación Básica } \\
\text { Mención Lengua Castellana y } \\
\text { Comunicación. } \\
\text { Pedagogía en Educación Básica } \\
\text { Mención Matemática. }\end{array}$ & $\begin{array}{l}\text { Aprendizaje Inicial } \\
\text { Efectivo en Ciencias } \\
\text { Naturales y Ciencias } \\
\text { Sociales. }\end{array}$ \\
\hline 17 & $\begin{array}{c}\text { Universidad de } \\
\text { Atacama }\end{array}$ & $\begin{array}{l}\text { Pedagogía en Educación } \\
\text { General Básica. }\end{array}$ & $\begin{array}{l}\text { Metodología de las } \\
\text { Ciencias Naturales. }\end{array}$ \\
\hline 18 & $\begin{array}{c}\text { Universidad del } \\
\text { Bío-Bío }\end{array}$ & $\begin{array}{l}\text { Pedagogía Educación General } \\
\text { Básica. }\end{array}$ & $\begin{array}{l}\text { Comprensión del } \\
\text { Medio Natural, Social } \\
\text { y Cultural. } \\
\text { Estudio y } \\
\text { Comprensión de la } \\
\text { Naturaleza. } \\
\text { Didáctica de las } \\
\text { Ciencias Naturales. } \\
\end{array}$ \\
\hline
\end{tabular}

\begin{tabular}{|c|c|c|c|}
\hline 19 & $\begin{array}{l}\text { Universidad de la } \\
\text { Frontera }\end{array}$ & & \\
\hline 20 & $\begin{array}{l}\text { Universidad de } \\
\text { Los Lagos }\end{array}$ & & \\
\hline 21 & $\begin{array}{l}\text { Universidad de } \\
\text { Magallanes }\end{array}$ & $\begin{array}{l}\text { Pedagogía en Educación } \\
\text { Básica. }\end{array}$ & $\begin{array}{l}\text { - Ciencias Naturales. } \\
\text { - Didáctica de las } \\
\text { Ciencias Naturales. }\end{array}$ \\
\hline 22 & $\begin{array}{l}\text { Universidad de } \\
\text { Talca }\end{array}$ & $\begin{array}{l}\text { Pedagogía en Educación } \\
\text { General Básica con Mención en } \\
\text { Alemán. }\end{array}$ & $\begin{array}{l}\text { - No se evidencia } \\
\text { asignatura relativa la } \\
\text { EA. }\end{array}$ \\
\hline
\end{tabular}




\begin{tabular}{|c|c|c|c|}
\hline $\mathbf{N}^{\circ}$ & UNIVERSIDAD & CARRERA & ASIGNATURAS \\
\hline 23 & $\begin{array}{c}\text { Universidad } \\
\text { Católica del Maule }\end{array}$ & $\begin{array}{l}\text { Pedagogía en Educación } \\
\text { General Básica. }\end{array}$ & $\begin{array}{l}\text { - El Proceso de } \\
\text { Indagación e } \\
\text { Investigación de } \\
\text { la Naturaleza y la } \\
\text { Sociedad y sus } \\
\text { Estrategias en el } \\
\text { Primer Ciclo de } \\
\text { Enseñanza Básica. } \\
\text { - Estrategias de las } \\
\text { Ciencias Naturales, } \\
\text { con Escolares de } \\
\text { Segundo Ciclo de } \\
\text { Enseñanza Básica. }\end{array}$ \\
\hline 24 & $\begin{array}{l}\text { Universidad } \\
\text { Católica de } \\
\text { la Santísima } \\
\text { Concepción }\end{array}$ & $\begin{array}{l}\text { Profesor de Educación Básica } \\
\text { con Mención en Lenguaje, } \\
\text { Comunicación y Ciencias } \\
\text { Sociales. } \\
\text { Profesor de Educación Básica } \\
\text { con Mención en Educación } \\
\text { Matemática y Ciencias } \\
\text { Naturales. } \\
\text { Profesor de Educación Básica } \\
\text { con Mención en Inglés. }\end{array}$ & $\begin{array}{l}\text { - Ciencias Integradas II } \\
\text { y su didáctica. }\end{array}$ \\
\hline 25 & $\begin{array}{c}\text { Universidad } \\
\text { Católica de Temuco }\end{array}$ & $\begin{array}{l}\text { Profesor de Educación Básica } \\
\text { con Mención en Lenguaje y } \\
\text { Comunicación. } \\
\text { Profesor de Educación Básica } \\
\text { con Mención en Matemática. } \\
\text { Profesor de Educación Básica } \\
\text { con Mención en Ciencias } \\
\text { Naturales. } \\
\text { Profesor de Educación Básica } \\
\text { con Mención en Historia, } \\
\text { Geografía y Ciencias Sociales. }\end{array}$ & $\begin{array}{l}\text { Ecología y Medio } \\
\text { Ambiente para la } \\
\text { Enseñanza. }\end{array}$ \\
\hline
\end{tabular}

Fuente: Elaboración de los autores. 


\section{Conclusión}

Según los antecedentes revisados y analizados, se evidencia una falta de concordancia o sintonía entre las propuestas curriculares de los proyectos formativos en las carreras de pedagogía y los planteamientos de las Bases Curriculares del Ministerio de Educación de Chile, toda vez que desde el currículum existe un abordaje somero y descontextualizado de las necesidades que demanda el medio en términos de formación.

En ese sentido, se hace necesaria una revisión desde el punto de vista de la tributación de las asignaturas cursadas en pregrado que permita conciliar la propuesta formativa con las necesidades del entorno, para ello, el tratamiento interdisciplinar y sistémico de la temática ambiental debe surgir a partir del diálogo entre las propias asignaturas componentes del flujo curricular que permitirán, de alguna forma, integrar saberes y alcanzar esta formación integral que propone la EA. 


\section{REFERENCIAS BIBLIOGRÁFICAS}

ALEA, A. (2005). "Breve historia de la educación ambiental: del conservacionismo hacia el desarrollo sostenible". Revista Futuros, 12(3), Recuperado de http://www. revistafuturos.info/futuros_12/hist_ea.htm

CALDERÓN, R.; SUMARÁN, R.; CHUMPITAZ, J. Y CAMPOS, J. (2011). Educación Ambiental. Aplicando el enfoque ambiental hacia una educación para el desarrollo sostenible. Huánuco: Kike.

CASTILLO, F. (2011). Las actividades en la naturaleza en la formación de profesores. Una aproximación desde el sentido. Saarbrücken: Editorial Académica Española.

CASTILLO, F. Y ALMONACID, A. (2012). "Las actividades en la naturaleza en la formación de profesores: un acercamiento desde los sentidos". Revista Educação e Pesquisa, 38(3), 667-681. Doi: http://dx.doi.org/10.1590/S151797022012005000012

CASTILLO, F. Y FUENTEALBA, M. (2014). Compendio de buenas prácticas ambientales. Talca: Universidad Católica del Maule.

CASTILLO, S. (2011). Concepciones y prácticas que presentan docentes de primer ciclo básico sobre la Educación Ambiental. Santiago: Universidad Academia de Humanismo Cristiano.

CHILE (1990). Ley 18.962. Ley Orgánica Constitucional de Enseñanza, del 07 de marzo de 1990 y última versión del 27 de septiembre de 2005. Biblioteca del Congreso Nacional de Chile. Recuperado de https://www.leychile.cl/ Navegar?idNorma $=30330$

(1994). Ley 19.300. Bases Generales del Medio Ambiente, del 01 de marzo de 1994 y última versión del 01 de junio de 2016. Biblioteca del Congreso Nacional de Chile. Recuperado de https://www.leychile.cl/Navegar?idNorma=30667 
(1996). Decreto Supremo $N^{\circ}$ 40, del 24 de enero de 1996 y última versión del 03 de marzo de 2016, que establece objetivos fundamentales y contenidos mínimos obligatorios de la educación básica y fija normas generales para su aplicación. Biblioteca del Congreso Nacional de Chile. Recuperado de https:// www.leychile.cl/Navegar?idNorma $=8043$

(2009). Política Nacional de Educación para el Desarrollo Sustentable. Santiago: Ministerio del Medio Ambiente. Recuperado de https://biblioteca.digital. gob.cl/handle/123456789/1406

(2009). Bases Curriculares de primero a sexto básico. Santiago: Ministerio de Educación de Chile.

(2018). Constitución Política de la República de Chile, del 17 de septiembre de 2005 y última versión del 16 de junio de 2018, que fija el texto refundido, coordinado y sistematizado de la Constitución Política de la República de Chile. Biblioteca del Congreso Nacional de Chile. Recuperado de https://www.leychile.cl/ Navegar?idNorma $=242302$

ECHARRI, F. (2007). Aprendizaje significativo y educación ambiental: Aplicaciones didácticas del Museo de Ciencias Naturales de la Universidad de Navarra. Navarra: España.

ENGEL, T. Y TOLFO, D. (2009). Métodos de pesquisa. Universidade Federal do Rio Grande do Sul: Porto Alegre.

FUENTEALBA, M. Y SOTO, L. (2016). "Valoración actitudinal frente a temas ambientales". Revista Luna Azul, №43, 448-467. Doi: http://dx.doi.org/10.17151/ luaz.2016.43.19

FUENTEALBA, M., MARÍN, F., CASTILLO, F. Y ROCO, L. (2017). "Análisis de la experiencia pedagógica: Campamento EXPLORA Chile VA! Valorando la biodiversidad maulina". Revista Actualidades Investigativas en Educación, 17(1), 229-254. Doi: http://dx.doi.org/10.15517/aie.v17i1.27211

GONZÁLEZ, M. (1996). "Principales tendencias y modelos de la educación ambiental en el sistema escolar". Revista Iberoamericana de Educación, № 11. Recuperado de http://www.rieoei.org/oeivirt/rie11a01.htm 
LAGOS, D. (2005). Tesis: Tendencia en los objetivos de los programas de Educación Ambiental en Chile entre los años 1994 al 2002, Universidad de Chile, Santiago: Chile.

MARTínEZ, J. (1999). Manual de Educación Ambiental. Unesco Etxea, Iberdrola: Bilbao.

MARTIN, F. (1996). Educación ambiental. Síntesis S.A.: Madrid.

MUÑOZ-PEDREROS, A. (2014). "La Educación Ambiental en Chile, una tarea aún pendiente". Revista Ambiente \& Sociedade, 17(3), 177-198. Doi: http://dx.doi. org/10.1590/S1414-753X2014000300011

OMEGNA, G. (2003). El camino recorrido por la Educación Ambiental. Revista Educación Ambiental, 1(1), 5-7.

PICÓN, L. Y MENDOZA, J. (2011). Aplicando el enfoque ambiental hacia una educación para el desarrollo sostenible. Actas del I Congreso Regional de Educación Ambiental. Huánuco: Perú.

SILVA, E. Y MUSZKAT, E. (2005). Metodologia da pesquisa e elaboração de dissertação. Florianópolis: Universidade Federal de Santa Catarina.

UNESCO. (1973). Informe de la Conferencia de las Naciones Unidas sobre el Medio Humano, Estocolmo, 5 a 16 de junio de 1972, Naciones Unidas, Nueva York.

(1978). Informe final Tbilisi 1977. Conferencia Intergubernamental sobre Educación Ambiental, Paris. Recuperado de http://unesdoc.unesco.org/ images/0003/000327/032763sb.pdf

(1980). Educación Ambiental. Las grandes orientaciones de la Conferencia de Tbilisi. Paris, Francia. 